\title{
Alternatives to the electron density for describing Coulomb systems
}

\author{
Paul W. Ayers ${ }^{\mathrm{a})}$ \\ Department of Chemistry, McMaster University, Hamilton, Ontario, L8S 4M1, Canada \\ Agnes Nagy \\ Department of Theoretical Physics, University of Debrecen, H-4010 Debrecen, Hungary \\ and Atomic and Molecular Physics Research Group, Hungarian Academy of Sciences, \\ H-4010 Debrecen, Hungary
}

(Received 16 January 2007; accepted 28 February 2007; published online 12 April 2007)

\begin{abstract}
Stimulated by the difficulty of deriving effective kinetic energy functionals of the electron density, the authors consider using the local kinetic energy as the fundamental descriptor for molecular systems. In this ansatz, the electron density must be expressed as a functional of the local kinetic energy. There are similar results for other quantities, including the local temperature and the Kohn-Sham potential. One potential advantage of these approaches-and especially the approach based on the local temperature-is the chemical relevance of the fundamental descriptor. (C) 2007 American Institute of Physics. [DOI: 10.1063/1.2718950]
\end{abstract}

\section{MOTIVATION}

Although the Kohn-Sham approach to density-functional theory (DFT) is now well established it bears remembering that almost 40 years separate the seminal papers of Thomas and Fermi from the breakthrough of Kohn and Sham. ${ }^{1-3}$ Even with the rise of the Kohn-Sham approach, "orbitalfree" computational techniques have never entirely vanished from the scene because orbital-free DFT is much faster than conventional Kohn-Sham calculations. In orbital-free DFT one only varies a single function of the three spatial coordinates (the electron density) instead of using $N$ threecoordinate functions (orbitals) or one six-coordinate function (the density matrix). ( $N$ is the number of electrons.) At present, however, orbital-free calculations are not really more "efficient" than Kohn-Sham calculations because while orbital-free calculations are computationally inexpensive, they are also very inaccurate. The problem is that the approximate kinetic energy functionals are ordinarily inadequate for describing bond breaking and other chemical processes in molecular systems. [Orbital-free methods have had more success in solid state materials, although seemingly only in cases where the electron density is low (most of the electrons are treated with a pseudopotential). ${ }^{4}$ ] Kohn-Sham theory circumvents the problem of evaluating the kinetic energy directly: instead the electron density is used to evaluate the Kohn-Sham potential, which is used to evaluate the Kohn-Sham orbitals, which are then used to evaluate the kinetic energy of the reference system of noninteracting electrons,

$$
T_{s}[\rho]=\sum_{i} n_{i}\left\langle\phi_{i}\left[v_{\mathrm{KS}}[\rho]\right]\left|\frac{-\nabla^{2}}{2}\right| \phi_{i}\left[v_{\mathrm{KS}}[\rho]\right]\right\rangle .
$$

Although $T_{s}[\rho]$ is usually slightly smaller than the true kinetic energy, the Kohn-Sham kinetic energy functional is $N$

${ }^{a)}$ Electronic mail: ayers@mcmaster.ca representable by construction and, as such, avoids the "variational catastrophes" that afflict ordinary orbital-free DFT. ${ }^{5-7}$

The 80 years that has elapsed since the original paper of Thomas testifies to the fact that expressing the kinetic energy as a functional of the electron density is extraordinarily difficult. This raises the question: might it be easier to express the electron density as a functional of the local kinetic energy or another similar quantity-like the local temperature or the Kohn-Sham potential-that determines the kinetic energy? We do not yet know whether or not it is easier to express the electron density as a functional of these descriptors, but we can establish that it is possible. This is the purpose of this paper.

- The local kinetic energy determines every property of a molecule, including its electron density. (See Sec. II for details and restrictions.)

- The local temperature determines every property of a Coulomb system, including its electron density. (See Sec. III for details and restrictions.)

- The Kohn-Sham effective potential determines every property of a Coulomb system, including its electron density. (See Sec. IV for details and restrictions.)

Because the Kohn-Sham potential can be determined (up to a trivial additive constant) from any single Kohn-Sham orbital

$$
v_{\mathrm{KS}}(\boldsymbol{r})=\frac{\left(\nabla^{2} / 2\right) \phi_{i}(\boldsymbol{r})}{\phi_{i}(\boldsymbol{r})}
$$

this last result implies that any single Kohn-Sham orbitaloccupied or unoccupied-determines the total electron density of any Coulomb system. (This extends the known result, which states that the frontier Kohn-Sham orbitals determine the total electron density and the density matrix. ${ }^{8,9}$ ) 
One can add to these results the recent proof:

- The shape function (the density per particle) determines every property of a Coulomb system, including its electron density. ${ }^{10}$

This paper is patterned after that result, which seems to be of conceptual $^{11-15}$ (and perhaps even computational ${ }^{16,17}$ ) utility as an alternative formulation of density-functional theory. This general sort of result can also be viewed as a "generalized density-functional theory," in which some other function replaces the electron density as the fundamental descriptor of molecular systems. ${ }^{18-20}$

It is clear from the preceding summary of our main results that this paper focuses on Coulomb systems, that is, systems where the external potential is due to a collection of point charges,

$$
v(\boldsymbol{r})=\sum_{\eta} \frac{-q_{\eta}}{\left|\boldsymbol{r}-\boldsymbol{R}_{\eta}\right|} .
$$

Molecules arise as the special case where all the point charges are positive integers. This class of systems is quite general, however. For any external potential that has only simple poles, one can construct the charge density,

$$
q_{v}(\boldsymbol{r})=\frac{-1}{4 \pi} \nabla^{2} v(\boldsymbol{r}),
$$

generating that external potential. Approximating this charge density using a numerical integration method automatically leads to a result with the desired form,

$$
\begin{aligned}
v(\boldsymbol{r})=\int \frac{q_{v}(\boldsymbol{R})}{|\boldsymbol{r}-\boldsymbol{R}|} d \boldsymbol{R} & \approx \sum_{\eta} \frac{w_{\eta} q_{v}\left(\boldsymbol{R}_{\eta}\right)}{\left|\boldsymbol{r}-\boldsymbol{R}_{\eta}\right|} \\
& =\sum_{\eta} \frac{\left(-w_{\eta} / 4 \pi\right)\left(\nabla^{2} v\left(\boldsymbol{R}_{\eta}\right)\right)}{\left|\boldsymbol{r}-\boldsymbol{R}_{\eta}\right|} .
\end{aligned}
$$

This implies that for any external potential that is not too singular, the effects of that external potential can be accurately approximated by a collection of point charges. Although our primary interest is in molecular electronic structure, the ability to accurately approximate the effects of almost any external potential using point charges suggests that our results have broad relevance.

\section{LOCAL KINETIC ENERGY AS A DESCRIPTOR OF MOLECULAR SYSTEMS}

The most general expression for the local kinetic energy employs the quasiprobability distribution $f(\boldsymbol{r}, \boldsymbol{p})$ for observing an electron at $\boldsymbol{r}$ with momentum $\boldsymbol{p}$. Specifically,

$$
t(\boldsymbol{r})=\int \frac{1}{2} \boldsymbol{p}^{2} f(\boldsymbol{r}, \boldsymbol{p}) d \boldsymbol{p} .
$$

However, because there are many ways to define $f(\boldsymbol{r}, \boldsymbol{p})$, this does not fully specify the local kinetic energy. ${ }^{21,22}$ Recommended by its simplicity and conceptual clarity is the positive semidefinite form ${ }^{23}$

$$
t_{+}\left(\boldsymbol{r}_{1}\right)=N \iint \ldots \int \frac{\left|\nabla_{\boldsymbol{r}_{1}} \Psi\left(\boldsymbol{r}_{1}, \boldsymbol{r}_{2} \ldots \boldsymbol{r}_{N}\right)\right|^{2}}{2} d \boldsymbol{r}_{2} d \boldsymbol{r}_{3} \ldots d \boldsymbol{r}_{N}
$$

More generally, Cohen has shown that the entire family of kinetic energy densities,

$$
t_{\alpha}(\boldsymbol{r})=t_{+}(\boldsymbol{r})+\alpha \nabla^{2} \rho(\boldsymbol{r})
$$

come from the same simple family of quasiprobability distributions. $^{22}$

Near a point charge $t_{+}(\boldsymbol{r})$ and $\rho(\boldsymbol{r})$ are composed of contributions from orbitals with the exponential dependence $e^{-Z r}$, where $r$ is the distance from the point charge being considered. As long as $\alpha \neq 0$, the form of $t_{\alpha}(\boldsymbol{r})$ near the point charge is dominated by a singularity from the Laplacian contribution,

$$
\begin{aligned}
t_{\alpha}(\boldsymbol{r}) & \sim \alpha \rho(0) \nabla^{2} e^{-2 Z r} \\
& =\alpha \rho(0) \frac{1}{r^{2}} \frac{\partial}{\partial r} r^{2} \frac{\partial}{\partial r} e^{-2 Z r} \\
r & \rightarrow \infty \\
& =-\alpha \frac{4 Z \rho(0)}{r}+4 \alpha Z^{2} \rho(0) .
\end{aligned}
$$

Far from a molecule, all of the natural orbitals have a characteristic exponential decay, $e^{-r \sqrt{2 \mathrm{IP}}}$, determined by the ionization potential of the system. The local kinetic energy has a similar exponential decay,

$$
t_{\alpha}(\boldsymbol{r}) \sim(1+8 \alpha) \mathrm{IP} e^{-2 \boldsymbol{r} \sqrt{2 \mathrm{IP}}}, \quad(r \rightarrow \infty) .
$$

The ionization potential of a system can be determined from the asymptotic decay of the local kinetic energy.

The main result from this section is that all of the properties of a neutral molecule in its equilibrium geometry can be determined from its local kinetic energy. Here we will use the local kinetic energy defined by Eqs. (7) and (8), with $\alpha$ $\neq 0$. To show this, we need to establish that (a) the local kinetic energy determines the location and type of atomic nuclei in a molecule and (b) the local kinetic energy determines the number of electrons in a molecule.

The reason we restricted ourselves to $\alpha \neq 0$ is because then the local kinetic energy has a simple pole at the location of the atomic nuclei,

$$
t_{\alpha}(\boldsymbol{r}) \sim \alpha \frac{-4 Z_{\eta} \rho\left(\boldsymbol{R}_{\eta}\right)}{\left|\boldsymbol{r}-\boldsymbol{R}_{\eta}\right|} .
$$

[Cf. Eq. (9).] If we knew the electron density at the atomic nuclei, $\rho\left(\boldsymbol{R}_{\eta}\right)$, then this equation would also suffice to determine the atomic number $Z_{\eta}$. While the electron density at an atomic nucleus usually changes slightly upon molecule formation, the "deformation" in the electron density upon forming a molecule is concentrated in the valence regions. ${ }^{24}$ The contribution to the electron density at the atomic nucleus is dominated by the core orbitals; these orbitals are essentially nonbonding in character and they are insensitive to the molecular environment. This suggests that we can approximate the electron density at the nucleus with the free atom density, i.e., 


$$
\rho\left(\boldsymbol{R}_{\eta}\right) \approx \rho_{Z_{\eta}}\left(\boldsymbol{R}_{\eta}\right) .
$$

Substituting the approximation in Eq. (12) into Eq. (11) should suffice to determine the atomic number for molecular kinetic energy densities. (A more elaborate treatment would seem to be required for molecular cations with extremely positive charges, but those problematic molecules are not expected to be stable. Similarly, the approximation in Eq. (12) will not be accurate near the united atom limit, but such molecules have extremely high energies.)

With the external potential now determined, the Schrödinger equation can be solved for $N=1,2, \ldots$ electrons. The ionization potentials

$$
\mathrm{IP}_{N}=E_{g . s .}[v, N-1]-E_{g . s .}[v, N]
$$

are then determined. The ionization potential is a strictly decreasing function of the number of electrons, so the number of electrons can be determined by finding the ionization potential that matches the characteristic asymptotic decay of the local kinetic energy.

Since the local kinetic energy determines both the external potential (through its characteristic singularities) and the number of electrons (from its asymptotic decay), the local kinetic energy suffices to determine all of the properties of a molecular system.

Further comment on the decreasing nature of the ionization potentials seems to be warranted. It has never been proven that the ionization potentials decrease as the number of electrons increase. This is an important open question in density-functional theory, since it bears on the convexity of the energy as a function of the number of electrons and therefore on the existence of the Legendre transform used to define the grand canonical ensemble. ${ }^{25}$ Several authors have advanced plausibility arguments for the decrease in the ionization potentials, ${ }^{10,25,26}$ and there is also some numerical evidence. $^{27,28}$ The result has so far resisted proof, however, even by extremely competent mathematicians. ${ }^{26}$ One reason this result is so hard to prove is that it is not generally true: for systems where the repulsion between particles is nonCoulomb (either hard-sphere-like ${ }^{26}$ or harmonic-oscillatorlike $\left.{ }^{29}\right)$, exceptions are known.

For atoms, one can make "plausibility" arguments for the decrease of the ionization potential with increasing electron number using, for example, the $N / Z$ expansion of March and White. Lieb and Perdew et al. noted that for systems of noninteracting fermions, the ionization potential never increases as the number of electrons increases. (For noninteracting electrons, the total energy is the sum of orbital energies, and no additional electron can occupy an orbital lower in energy than the previous electron. ${ }^{25,26}$ ) The result can also be motivated without the use of Fermi statistics. Adopting a simple model where the energy of an $N$-electron system is $N$ times the expectation value of the "one-electron operator," $\hat{h}(\boldsymbol{r})=-\nabla_{i}^{2} / 2+v\left(\boldsymbol{r}_{i}\right)$ plus $N(N-1) / 2$ times the expectation value of the "two-electron operator," $\hat{v}_{e e}=1 /\left|\boldsymbol{r}_{i}-\boldsymbol{r}_{j}\right|$, then the ionization potentials of the $N-k$ electron systems increase according to the formula $\operatorname{IP}_{N-k}=-\langle\hat{h}\rangle-(N-1-k)\left\langle\hat{v}_{e e}\right\rangle$. In this argument, convexity arises because the number of electronelectron repulsions grows quadratically with the number of electrons. (This rationalization would hold even for charged bosons. For fermions, there is an additional effect because the Pauli exclusion principle indicates that there will be at most two electrons in each orbital, and so the orbital energy of the most easily ionized electrons increases as the number of electrons increases.)

\section{LOCAL TEMPERATURE AS A DESCRIPTOR OF COULOMB SYSTEMS}

The local kinetic energy theory is not entirely satisfactory because it is restricted to molecular systems, that is, systems with external potentials from a collection of positive-integer point charges,

$$
v_{\mathrm{mol}}(\boldsymbol{r})=\sum_{\eta} \frac{-Z_{\eta}}{\left|\boldsymbol{r}-\boldsymbol{R}_{\eta}\right|}, \quad Z_{\eta}=1,2, \ldots
$$

We can generalize the theory to other Coulombic systems (where the point charges can be any real number)

$$
v_{\text {Coul }}(\boldsymbol{r})=\sum_{\eta} \frac{-q_{\eta}}{\left|\boldsymbol{r}-\boldsymbol{R}_{\eta}\right|}, \quad q_{\eta} \in \mathbb{R}
$$

by using the local temperature..$^{30,31}$

The local temperature was introduced to densityfunctional theory by Ghosh et al. in their reformulation of electronic density-functional theory into a formalism with the same structure as classical statistical mechanics. ${ }^{30,31}$ They defined the local temperature $T_{\alpha}(\boldsymbol{r})$ through the equation

$$
t_{\alpha}(\boldsymbol{r})=\rho(\boldsymbol{r})\left(\frac{3}{2} k_{B} T_{\alpha}(\boldsymbol{r})\right)=\rho(\boldsymbol{r})\left(\frac{3}{2} \theta_{\alpha}(\boldsymbol{r})\right)
$$

Here $t_{\alpha}(\boldsymbol{r})$ is the local kinetic energy from Eq. (8) and $\theta_{\alpha}(\boldsymbol{r})$ is the local temperature measured in units where the Boltzmann constant is unity. The local temperature can be used to elucidate the electronic structure of molecules. In particular, because localized electrons are comparatively "hot," the local temperature provides a measure of the "nighness" of the electrons in an electron pair. ${ }^{23,32}$

The local temperature determines all properties of Coulomb systems because the local temperature determines the external potential and the number of electrons for this type of system. To determine the external potential, the asymptotic form local temperature near a point charge is used to determine the position and the charge of the point charges in the external potential. The asymptotic decay of the local temperature is then used to determine the ionization potential, which is used to determine the number of electrons in the system.

We will now present this construction in more detail.

Step 1: Determine the locations of the point charges, $\left\{\boldsymbol{R}_{\eta}\right\}$. If $\alpha \neq 0$, then the point charges are located where $\theta_{\alpha \neq 0}(\boldsymbol{r})$ has a singularity.

Step 2: Determine the change on each point charge, $\left\{q_{\eta}\right\}$. The charges themselves are determined by the strength of the singularity, 


$$
\theta_{\alpha}(\boldsymbol{r}) \stackrel{\left|\boldsymbol{r}-\boldsymbol{R}_{\eta}\right| \rightarrow 0}{\sim} \alpha \frac{-8 q_{\eta}}{3\left|\boldsymbol{r}-\boldsymbol{R}_{\eta}\right|} .
$$

This follows from the cusp conditions on the local kinetic energy $^{33}$ and the cusp conditions on the electron density. ${ }^{34-36}$

Step 3: Determine the ionization potential. The ionization potential can be determined from the asymptotic value of the local temperature,

$$
t_{\alpha}(\boldsymbol{r}) \stackrel{r \rightarrow \infty}{\sim} \frac{2}{3}(1+8 \alpha) \text { IP. }
$$

Using the external potential determined in steps 1 and 2 and repeatedly solving the Schrödinger equation for $N$ $=1,2,3, \ldots$ electrons, one can determine the unique value of the ionization potential that corresponds to the system of interest.

A theory based on the local temperature (a local kinetic energy based treatment would be similar) would have the same cost advantages as orbital-free DFT, since the energy would be a (hopefully) simple expression with the form

$$
\begin{aligned}
E_{v}[t]= & \int \rho[\theta ; \boldsymbol{r}]\left(\frac{3}{2} \theta(\boldsymbol{r})+v(\boldsymbol{r})\right) d \boldsymbol{r}+J[\rho[\theta]] \\
& +V_{x c}[\rho[\theta], \theta] .
\end{aligned}
$$

The first term (kinetic energy) and second (electron-nuclear interaction energy) contributions to this expression are written using the density as a functional of the local temperature, $\rho[\theta ; \boldsymbol{r}]$. The third term is the classical Coulomb repulsion energy between the electrons,

$$
\frac{1}{2} \iint \frac{\rho(\boldsymbol{r}) \rho\left(\boldsymbol{r}^{\prime}\right)}{\left|\boldsymbol{r}-\boldsymbol{r}^{\prime}\right|} d \boldsymbol{r} d \boldsymbol{r}^{\prime} .
$$

The last term is the potential contribution to the exchangecorrelation energy $\left(V_{x c}=E_{x c}-T_{c}\right)$. (Notice that meta-GGAs (i.e., exchange-correlation energy functionals that are bifunctionals of the electron density and the local kinetic energy density) are very natural functionals in this approach! ${ }^{37-47}$ We can include the correlation-kinetic contribution to the energy in the kinetic-energy term of Eq. (19), include it in the exchange-correlation energy, or treat it separately.)

The proposed method assumes that it is easier to approximate the electron density as a functional of the local temperature, $\rho[\theta ; \boldsymbol{r}]$, than it is to approximate the local temperature as a functional of the electron density, $\theta[\rho ; \boldsymbol{r}]$. At this stage in our research, it is impossible to say whether this is true. Eighty years of failure at developing density functionals for the kinetic energy suggests, however, that it can scarcely be more difficult to obtain accurate approximations to $\rho[\theta ; \boldsymbol{r}]$.

\section{KOHN-SHAM POTENTIAL AS A DESCRIPTOR FOR COULOMB SYSTEMS}

As a final alternative, we consider using the Kohn-Sham potential

$$
v_{\mathrm{KS}}(\boldsymbol{r})=v(\boldsymbol{r})+v_{J}[\rho ; \boldsymbol{r}]+v_{x c}[\rho ; \boldsymbol{r}]
$$

as a descriptor for Coulomb systems. Neither the Coulomb potential

$$
v_{J}[\rho ; \boldsymbol{r}]=\int \frac{\rho\left(\boldsymbol{r}^{\prime}\right)}{\left|\boldsymbol{r}-\boldsymbol{r}^{\prime}\right|} d \boldsymbol{r}^{\prime}
$$

nor the exchange-correlation potential

$$
v_{x c}[\rho ; \boldsymbol{r}]=\frac{\delta E_{x c}[\rho]}{\delta \rho(\boldsymbol{r})}
$$

is singular at atomic nuclei. ${ }^{48,49}$ (More generally, it seems that neither function is singular except possibly where the electron density is singular, which never occurs in molecular systems.) Since the only singularities in the Kohn-Sham potential of a Coulomb system are those associated with the point charges in the external potential, the external potential is readily determined from the Kohn-Sham potential.

The number of electrons is also readily determined. First add up all of the point charges,

$$
q_{\mathrm{tot}}=\sum_{\eta} q_{\eta}
$$

Far from a molecule, the Kohn-Sham potential assumes the asymptotic form

$$
v_{\mathrm{KS}}(\boldsymbol{r}) \sim \frac{-q_{\mathrm{tot}}+N-1}{r} .
$$

The number of electrons is then readily expressed using a "sum rule" for the Kohn-Sham potential,

$$
N=\frac{-1}{4 \pi} \int \nabla^{2} v_{\mathrm{KS}}(\boldsymbol{r}) d \boldsymbol{r}+q_{\mathrm{tot}}+1 .
$$

[This sum rule is a simple consequence of Poisson's equation for the external potential and the Coulomb potential and the sum rule for the exchange-correlation potential. ${ }^{50-52}$ It is readily proved using Gauss's theorem and the asymptotic decay of the Kohn-Sham potential in Eq. (25).]

There is another way to prove this result. First use the Kohn-Sham potential to determine the number of electrons [Eq. (26)]. Then solve the Kohn-Sham equations and construct the electron density by occupying the $N$ lowest-energy Kohn-Sham spin orbitals,

$$
\rho(\boldsymbol{r})=\sum_{i=1}^{N}\left|\phi_{i}(\boldsymbol{r})\right|^{2} .
$$

All the observable properties are then determined by the Hohenberg-Kohn theorem. This result has an interesting formal implication. Given any Kohn-Sham potential, one can solve the Kohn-Sham equations and construct the electron density for $N=1,2,3, \ldots$ electrons. These electron densities are (assuming $v$-representability) ground-state electron densities for a sequence of external potentials, $v_{1}(\boldsymbol{r}), v_{2}(\boldsymbol{r}), v_{3}(\boldsymbol{r}), \ldots$ Our result indicates that at most one of these external potentials is Coulombic.

The fact that $v_{\mathrm{KS}}(\boldsymbol{r})$ can be used as a fundamental descriptor for Coulomb systems may have interesting implications in formal (and also practical) density-functional theory. For example, the importance of Kohn-Sham potential for describing Coulomb systems has recently been emphasized by Theophilou and co-workers, who expressed the Kohn-Sham potential as a functional of the external potential. ${ }^{53-55}[\mathrm{~A}$ 
(purely formal) link between the Kohn-Sham potential and the external potential can also be obtained directly from the adiabatic connection formulation in the potential representation. $\left.{ }^{56}\right]$

It may also be possible to design practical computational schemes in which $v_{\mathrm{KS}}(\boldsymbol{r})$ is the fundamental variable. One can evaluate the Kohn-Sham kinetic energy directly using the virial relation

$$
T_{s}\left[v_{\mathrm{KS}}\right]=\frac{1}{2} \int \rho\left[v_{\mathrm{KS}} ; \boldsymbol{r}\right] \boldsymbol{r} \cdot \nabla v_{\mathrm{KS}}(\boldsymbol{r}) d \boldsymbol{r} .
$$

This gives an explicit formula for the energy without reference to the Kohn-Sham orbitals,

$$
\begin{aligned}
E_{v}\left[v_{\mathrm{KS}}\right]= & T_{s}\left[v_{\mathrm{KS}}\right]+\int \rho\left[v_{\mathrm{KS}} ; \boldsymbol{r}\right] v(\boldsymbol{r}) d \boldsymbol{r}+J\left[\rho\left[v_{\mathrm{KS}}\right]\right] \\
& +E_{x c}\left[\rho\left[v_{\mathrm{KS}}\right]\right] .
\end{aligned}
$$

Analogous to the formulation based on the local temperature [cf. Eq. (19)], a functional for the electron density as a functional of the Kohn-Sham potential is needed.

One advantage of using the Kohn-Sham potential as the fundamental descriptor of Coulomb systems is that one already knows how to determine the electron density from the Kohn-Sham potential (just solve the Kohn-Sham equations!). If one does this, then Eq. (29) is just a reformulation of the conventional Kohn-Sham procedure. If one is seeking a method that is more efficient than the usual Kohn-Sham approach, then it might be more interesting to consider the Kohn-Sham potential in conjunction with the proposed Christoffel-Darboux formulas for the electron density.

Note that since any Kohn-Sham orbital determines the Kohn-Sham potential (just invert the Kohn-Sham equation), one could also use a single Kohn-Sham orbital as the descriptor of a Coulomb system.

\section{CONCLUDING REMARKS}

Over the last several years, these authors and others have considered a variety of approaches that might be classified as "generalized density-functional theories." Some of these theories seek to use not only the electron density but also additional information (values of certain properties, ${ }^{58-60}$ information about the pair density, ${ }^{20,61-64}$ information about the local kinetic energy (or equivalently, the local temperature), ${ }^{23,37,65}$ etc.) That class of generalized densityfunctional theories seeks to achieve greater accuracy at greater computational cost than the conventional Kohn-Sham approach. Other sorts of generalized density-functional theories are designed for cases where less accuracy would be acceptable, provided that the method was less computationally expensive than an ordinary Kohn-Sham calculation. In such cases one is interested in descriptors that contain less information than the full electron density (the density-perparticle (shape function), ${ }^{10}$ frontier orbitals, ${ }^{8,9}$ etc.) are used. Despite the large amount of recent work in this latter area, there do not seem to have been any major breakthroughs.
Perhaps the only way to accelerate Kohn-Sham calculations without unacceptable loss of accuracy is to use semiempirical approaches.

Nonetheless, these authors have not given up on this problem quite yet and the present paper is an effort in this direction. This paper provides theoretical "permission" for researchers to rewrite density functionals as functions of the local temperature, local kinetic energy density, or the KohnSham potential alone, without any explicit information about the number of electrons. Based on our previous work, one can also write functionals of the shape function ${ }^{10}$ or any Kohn-Sham orbital or orbital density (since the Kohn-Sham orbitals and orbital densities determine the Kohn-Sham potential). ${ }^{8}$ There are two conceivable advantages to these approaches. In some cases, it may be easier to write functionals in terms of one descriptor than another. (There is some evidence, for example, that the periodic trends in the atoms are more readily described by a descriptor that does not depend explicitly on the number of electrons. ${ }^{16}$ ) It is also advantageous when the fundamental descriptor of the system has clear chemical relevance. ${ }^{66}$ This, for example, is one of the reasons the frontier-orbital descriptor approach has attracted interest. ${ }^{8,9}$ The local kinetic energy is related to the width of the exchange-correlation hole ${ }^{67,68}$ and the local temperature is directly related to the "correlation length" between electron pairs. This means that the local temperature is a measure of nighness ${ }^{23}$ and can be used in the same way other "electron localization functions" are. ${ }^{67,69}$ If useful approximations to the energy can be written as functionals of the local temperature, then a "local-temperature functional theory" would possibly be preferable to conventional density-functional theory because the local temperatureunlike the electron density-provides direct access to the conceptually useful "Lewis structure" of electron pairs.

If one accepts this philosophy, then it will be interesting to attempt to prove analogous results for other functions of chemical relevance. For example, one might attempt to extend these results to the local ionization potential ${ }^{70}$ and the reactivity indicators associated with density-functional theory. ${ }^{71,72}$ As a first result along these lines, we can state that the Fukui functions ${ }^{73}$ determine all the properties of Coulomb systems. (This can be proved using the methods in this paper, along with known results for (a) the cusp conditions of the Fukui functions ${ }^{74}$ and (b) the characteristic asymptotic decay of the Fukui functions. ${ }^{74}$ )

Finally, we note that the results on the asymptotic form of the local kinetic energy density and the electron density near point charges ("cusp conditions") $10,33,75-77$ and far from the system ("decay conditions") $)^{10,78}$ can be generalized to excited states. For this reason, it may be possible to construct "excited-state" generalizations of DFT that use the characteristic features of, for example, the local temperature of excited states. Results of this type are known for the electron density $^{79-81}$ and the shape function, ${ }^{10}$ for example.

\section{ACKNOWLEDGMENTS}

One of the authors (P.W.A.) acknowledges financial support from NSERC and the Canada Research Chairs. 
Another author (A.N.) acknowledges support from OTKA Grant No. T042505.

${ }^{1}$ L. H. Thomas, Proc. Cambridge Philos. Soc. 23, 542 (1927).

${ }^{2}$ E. Fermi, Z. Phys. 48, 73 (1928).

${ }^{3}$ W. Kohn and L. J. Sham, Phys. Rev. 140, A1133 (1965).

${ }^{4}$ Y. A. Wang and E. A. Carter, Orbital-Free Kinetic-Energy Density Functional Theory, edited by S. D. Schwartz (Kluwer, Dordrecht, 2000), Chap. 5, pp. 117-184.

${ }^{5}$ P. W. Ayers and S. B. Liu, Phys. Rev. A 75, 022504 (2007).

${ }^{6}$ V. V. Karasiev, E. V. Ludena, and A. N. Artemyev, Phys. Rev. A 62, 062510 (2000).

${ }^{7}$ E. S. Kryachko and E. V. Ludena, Phys. Rev. A 43, 2179 (1991).

${ }^{8}$ P. W. Ayers, Theor. Chem. Acc. 110, 267 (2003).

${ }^{9}$ I. A. Howard, N. H. March, and P. W. Ayers, Chem. Phys. Lett. 385, 231 (2004).

${ }^{10}$ P. W. Ayers, Proc. Natl. Acad. Sci. U.S.A. 97, 1959 (2000).

${ }^{11}$ P. Geerlings, F. De Proft, and P. W. Ayers, Theor. Comput. Chem. 19, 1 (2007).

${ }^{12}$ F. De Proft, P. W. Ayers, K. D. Sen, and P. Geerlings, J. Chem. Phys. 120, 9969 (2004).

${ }^{13}$ K. D. Sen, F. De Proft, A. Borgoo, and P. Geerlings, Chem. Phys. Lett. 410, 70 (2005).

${ }^{14}$ A. Borgoo, M. Godefroid, K. D. Sen, F. De Proft, and P. Geerlings, Chem. Phys. Lett. 399, 363 (2004).

${ }^{15}$ P. Geerlings, G. Boon, C. Van Alsenoy, and F. De Proft, Int. J. Quantum Chem. 101, 722 (2005).

${ }^{16}$ P. W. Ayers, F. De Proft, and P. Geerlings, Phys. Rev. A 75, 012508 (2007).

${ }^{17}$ P. W. Ayers, Phys. Rev. A 71, 062506 (2005).

${ }^{18}$ T. K. Ng, Phys. Rev. B 44, 2407 (1991).

${ }^{19}$ P. W. Ayers, S. Golden, and M. Levy, J. Chem. Phys. 124, 054101 (2006).

${ }^{20}$ P. W. Ayers and M. Levy, J. Chem. Sci. 117, 507 (2005).

${ }^{21}$ L. Cohen, J. Chem. Phys. 80, 4277 (1984).

${ }^{22}$ L. Cohen, J. Chem. Phys. 70, 788 (1979).

${ }^{23}$ P. W. Ayers, R. G. Parr, and A. Nagy, Int. J. Quantum Chem. 90, 309 (2002).

${ }^{24}$ F. L. Hirshfeld, Theor. Chem. Acc. 44, 129 (1977).

${ }^{25}$ J. P. Perdew, R. G. Parr, M. Levy, and J. L. Balduz, Jr., Phys. Rev. Lett. 49, 1691 (1982)

${ }^{26}$ E. H. Lieb, Int. J. Quantum Chem. 24, 243 (1983).

${ }^{27}$ S. J. Chakravorty and E. R. Davidson, J. Phys. Chem. 100, 6167 (1996).

${ }^{28}$ S. J. Chakravorty, S. R. Gwaltney, E. R. Davidson, F. A. Parpia, and C. F. Fischer, Phys. Rev. A 47, 3649 (1993).

${ }^{29}$ P. Phillips and E. R. Davidson, The Chemical Potential of Interacting Fermions in a Harmonic Potential, edited by J. P. Dahl and J. Avery (Plenum, New York, 1984), pp. 43-52.

${ }^{30}$ S. K. Ghosh and M. Berkowitz, J. Chem. Phys. 83, 2976 (1985).

${ }^{31}$ S. K. Ghosh, M. Berkowitz, and R. G. Parr, Proc. Natl. Acad. Sci. U.S.A. 81, 8028 (1984)

${ }^{32}$ H. L. Schmider and A. D. Becke, THEOCHEM 527, 51 (2000).

${ }^{33}$ Z. Janosfalvi, K. D. Sen, and A. Nagy, Phys. Lett. A 344, 1 (2005).

${ }^{34}$ T. Kato, Commun. Pure Appl. Math. 10, 151 (1957).

${ }^{35}$ R. T. Pack and W. B. Brown, J. Chem. Phys. 45, 556 (1966).
${ }^{36}$ E. Steiner, J. Chem. Phys. 39, 2365 (1963).

${ }^{37}$ S. K. Ghosh and R. G. Parr, Phys. Rev. A 34, 785 (1986).

${ }^{38}$ R. G. Parr, K. Rupnik, and S. K. Ghosh, Phys. Rev. Lett. 56, 1555 (1986).

${ }^{39}$ W. Yang, R. G. Parr, and C. Lee, Phys. Rev. A 34, 4586 (1986).

${ }^{40}$ C. Lee, W. Yang, and R. G. Parr, Phys. Rev. B 37, 785 (1988).

${ }^{41}$ S. N. Maximoff and G. E. Scuseria, J. Chem. Phys. 114, 10591 (2001).

${ }^{42}$ J. P. Perdew, S. Kurth, A. Zupan, and P. Blaha, Phys. Rev. Lett. 82, 2544 (1999).

${ }^{43}$ A. D. Becke, Int. J. Quantum Chem. 23, 1915 (1983).

${ }^{44}$ A. D. Becke, J. Comput. Chem. 20, 63 (1999).

${ }^{45}$ A. D. Becke, Int. J. Quantum Chem. S28, 625 (1994).

${ }^{46}$ T. Van Voorhis and G. E. Scuseria, J. Chem. Phys. 109, 400 (1998).

${ }^{47}$ T. Van Voorhis and G. E. Scuseria, Mol. Phys. 92, 601 (1997).

${ }^{48}$ Z. X. Qian and V. Sahni, Int. J. Quantum Chem. 80, 555 (2000).

${ }^{49}$ Z. X. Qian and V. Sahni, Int. J. Quantum Chem. 79, 205 (2000).

${ }^{50}$ S. B. Liu, P. W. Ayers, and R. G. Parr, J. Chem. Phys. 111, 6197 (1999).

${ }^{51}$ P. W. Ayers and M. Levy, J. Chem. Phys. 115, 4438 (2001).

${ }^{52}$ A. Gorling, Phys. Rev. Lett. 83, 5459 (1999).

${ }^{53}$ A. K. Theophilou and V. Glushkov, Int. J. Quantum Chem. 104, 538 (2005).

${ }^{54}$ A. K. Theophilou and V. N. Glushkov, J. Chem. Phys. 124, 034105 (2006).

${ }^{55}$ S. Thanos and A. K. Theophilou, J. Chem. Phys. 124, 204109 (2006).

${ }^{56}$ W. T. Yang, P. W. Ayers, and Q. Wu, Phys. Rev. Lett. 92, 146404 (2004).

${ }^{57}$ K. A. Dawson and N. H. March, Phys. Lett. A 106, 161 (1984).

${ }^{58}$ X. Y. Pan, V. Sahni, and L. Massa, Phys. Rev. Lett. 93, 130401 (2004).

${ }^{59}$ K. Higuchi and M. Higuchi, Phys. Rev. B 69, 165118 (2004).

${ }^{60}$ M. Higuchi and K. Higuchi, Phys. Rev. B 69, 035113 (2004).

${ }^{61}$ P. Ziesche, Phys. Lett. A 195, 213 (1994).

${ }^{62}$ P. W. Ayers, J. Math. Phys. 46, 062107 (2005).

${ }^{63}$ A. Nagy, Phys. Rev. A 66, 022505 (2002).

${ }^{64}$ P. W. Ayers, Phys. Rev. A 74, 042502 (2006).

${ }^{65}$ C. Lee and R. G. Parr, Phys. Rev. A 35, 2377 (1987).

${ }^{66}$ P. W. Ayers, Chem. Phys. Lett. 438, 148 (2007).

${ }^{67}$ A. D. Becke and K. E. Edgecombe, J. Chem. Phys. 92, 5397 (1990).

${ }^{68}$ M. Berkowitz, Chem. Phys. Lett. 129, 486 (1986).

${ }^{69}$ P. W. Ayers, J. Chem. Sci. 117, 441 (2005).

${ }^{70}$ P. Sjoberg, J. S. Murray, T. Brinck, and P. Politzer, Can. J. Chem. 68, 1440 (1990).

${ }^{71}$ P. Geerlings, F. De Proft, and W. Langenaeker, Chem. Rev. (Washington, D.C.) 103, 1793 (2003).

${ }^{72}$ P. W. Ayers, J. S. M. Anderson, and L. J. Bartolotti, Int. J. Quantum Chem. 101, 520 (2005).

${ }^{73}$ R. G. Parr and W. Yang, J. Am. Chem. Soc. 106, 4049 (1984).

${ }^{74}$ P. W. Ayers and M. Levy, Theor. Chem. Acc. 103, 353 (2000).

${ }^{75}$ A. Nagy and K. D. Sen, J. Chem. Phys. 115, 6300 (2001).

${ }^{76}$ A. Nagy and K. D. Sen, Chem. Phys. Lett. 332, 154 (2000).

${ }^{77}$ A. Nagy and K. D. Sen, J. Phys. B 33, 1745 (2000).

${ }^{78}$ J. Katriel and E. R. Davidson, Proc. Natl. Acad. Sci. U.S.A. 77, 4403 (1980).

${ }^{79}$ A. Nagy, Adv. Quantum Chem. 42, 363 (2003).

${ }^{80}$ A. Nagy, Int. J. Quantum Chem. 70, 681 (1998).

${ }^{81}$ P. W. Ayers, M. Levy, and A. Nagy (unpublished). 